\title{
Vector Measure Orthonormal Systems and Self-weighted Functions Approximation
}

\author{
By \\ Luis M. García-Raffi, Damián Ginestar and Enrique A. SÁnchez PÉrez*
}

\begin{abstract}
If $\lambda$ is a positive vector measure on $l_{2}$, the notion of $\lambda$-orthonormal system of functions leads to a natural generalization of the relation between orthogonality and best approximation in Hilbert spaces for spaces $L_{2}(\lambda)$ of square integrable functions with respect to $\lambda$. We provide a vector orthogonality criterion that induces the definition of a particular projection on a subspace of $L_{2}(\lambda)$ that we call the selfweighted approximation. As an application, we show a new extrapolation technique.
\end{abstract}

\section{$\S 1 . \quad$ Introduction and Basic Results}

The properties of the spaces of integrable functions with respect to a vector measure have been studied in both abstract and applied contexts (see for example $[1,4,11,3,10,13])$. Following the investigation about applications of vector measure integration and the spaces of integrable functions with respect to vector measures (see $[3,10,11,13])$, we propose in this paper a general framework for function approximation. Our aim is to extend the notion of orthogonal projection of a function on a subspace of a Hilbert space to the context of the spaces $L_{2}(\lambda)$ of square integrable functions with respect to a vector measure $\lambda$ in order to obtain new fitting procedures. Thus, Section 1 is devoted to introduce several definitions involving generalized orthonormal sequences and to obtain the main results on approximation with respect to

Communicated by H. Okamoto. Received November 25, 2003. Revised April 5, 2004. 2000 Mathematics Subject Classification(s): 46E30, 46G10, 41A30.

Key words: Vector measures, orthogonal functions, approximation.

The first and third authors acknowledge the support of the Spanish Ministry of Science and Technology, Plan Nacional I+D+I, grant BFM2003-02302

* Departamento de Matemática Aplicada. Camino de Vera. 46071 Valencia. Spain.

e-mail: Imgarcia@mat.upv.es, dginesta@mat.upv.es, easancpe@mat.upv.es 
what we call the $\mathcal{C}$-orthogonal distance. We start providing the fundamental definitions and results on integration with respect to a vector measure, to center our attention in the case of positive countably additive vector measures with values in $l_{2}$, that will be our framework. Theorems 1.1 and 1.2 give the tools to construct what we call the self-weighted approximation in $L_{2}(\lambda)$.

In Section 2 we introduce the notion of conic vector measure with values in $l_{2}$, and we apply the results of Section 1 to give a geometrical meaning to the self-weighted approximation. Finally, we develop two examples and we show that our results would be applied as an extrapolation technique.

Let us introduce some basic definitions and results on vector measure integration theory. Let $X$ be a Banach space. We will denote by $X^{\prime}$ its dual space and by $B_{X}$ its unit ball. Let $(\Omega, \Sigma)$ be a measurable space. Consider a countably additive vector measure $\lambda: \Sigma \rightarrow X$. We say that a measurable real function $f: \Omega \rightarrow R$ is integrable with respect to $\lambda$ ( $\lambda$-integrable for short) if it is scalarly integrable (i.e. is integrable with respect to each scalar measure given by $\left.\lambda_{x^{\prime}}(A):=\left\langle\lambda(A), x^{\prime}\right\rangle, A \in \Sigma, x^{\prime} \in X^{\prime}\right)$ and there is an element denoted by $\int_{A} f d \lambda \in X$ for every $A \in \Sigma$ such that $\left\langle\int_{A} f d \lambda, x^{\prime}\right\rangle=\int_{A} f d \lambda_{x^{\prime}}$ (see [6] or [7]). If $1<p<\infty$, we say that a measurable real function $f$ is $p \lambda$-integrable if $|f|^{p}$ is $\lambda$-integrable. In the case $p=2$, we say that $f$ is square $\lambda$-integrable.

The Köthe function space of (classes of) $p \lambda$-integrable functions is denoted by $L_{p}(\lambda)$. An exhaustive study of the properties of this space when $p=1$ can be found in [1]. The main properties of the integration operator $I: L_{1}(\lambda) \rightarrow X$ defined by $I(f):=\int_{\Omega} f d \lambda$ have been studied in [9]. For the case $1<p<\infty$ the reader can find the proof of several basic Banach lattice properties in [11]. In particular it can be found in this paper the proof of the fact that the pointwise product $f g$ of a function $f \in L_{p}(\lambda)$ and a function $g \in L_{p^{\prime}}(\lambda)$ is $\lambda$-integrable. In the particular case of a space $L_{2}(\lambda)$ it is possible to define the notion of $\lambda$ orthogonality (see [3] and [13]). Two functions $f, g \in L_{2}(\lambda)$ are $\lambda$-orthogonal if $\int_{\Omega} f g d \lambda=0$. It is interesting to remark that $L_{2}(\lambda)$ is not in general isomorphic to a Hilbert space (see $[13,10]$ ).

In this paper we mainly deal with positive vector measures, i.e. countably additive vector measures taking values in positive cones of Banach lattices. In fact, we center our attention in the case $X=l_{2}$, the Hilbert space of sequences of (real) numbers endowed with the usual order: if $\left(x_{i}\right)_{i=1}^{\infty},\left(y_{i}\right)_{i=1}^{\infty} \in l_{2}$, $\left(x_{i}\right)_{i=1}^{\infty} \leq\left(y_{i}\right)_{i=1}^{\infty}$ if and only if $x_{i} \leq y_{i}$ for every natural number $i$. Thus, the positive cone of this space is the set $l_{2}^{+}=\left\{\left(x_{i}\right)_{i=1}^{\infty} \in l_{2} \mid x_{i} \geq 0, i \in N\right\}$, where $N$ is the set of natural numbers. If $n \in N$, we will use also the notation $l_{2}^{n}$ for the 
$n$-dimensional $R^{n}$ space with the Euclidean norm. We will simply write $\|\cdot\|$ for the norm of $l_{2}$.

We will use standard concepts and notation of the Banach space theory. The reader can find all the results that are needed about function spaces in [5] and [8], and the answer to general questions on vector measure theory in [2]. If $\nu$ is a scalar measure, we will write $|\nu|$ for its variation. If $\lambda$ is a vector measure on a Banach space $X$, we will say that a positive scalar measure $\mu$ controls $\lambda$ if $\mu(A)=0$ implies $\lambda(A)=0$ for every $A \in \Sigma$. Consider and element $x^{\prime} \in B_{X^{\prime}}$. We will say that the measure $\left|\lambda_{x^{\prime}}\right|$ is a Rybakov measure for $\lambda$ if it controls $\lambda$. It is well known that we can always find a Rybakov measure for any vector measure $\lambda$ (see for example [2]). If $x \in l_{2}$, we will write $\langle x\rangle$ for the linear span of $x$. However, if $B=\left\{h_{i} \mid i \in I\right\}$ is a family of functions we will use the symbol $\operatorname{span} B$ to denote the linear span of $B$. We will denote by $R$ the set of all real numbers, and by $e_{i}, i \in N$, the elements of the canonical basis of $l_{2}$.

The following proposition establishes several basic facts on the structure of the spaces of square $\lambda$-integrable functions that will be useful through the paper.

Proposition 1.1. $\quad$ Let $X$ be a Banach space. Let $\lambda: \Sigma \rightarrow X$ be a vector measure. Then:

1) If $\mu$ is a Rybakov measure for $\lambda$, the set of all the (equivalence classes of $\mu$-a.e. equal) square $\lambda$-integrable functions defines the Köthe function space $L_{2}(\lambda)$ (over $\left.\mu\right)$ with the norm $\|f\|_{L_{2}(\lambda)}:=\sup _{x^{\prime} \in B_{X^{\prime}}}\left(\int_{\Omega} f^{2} d\left|\lambda_{x^{\prime}}\right|\right)^{\frac{1}{2}}$, $f \in L_{2}(\lambda)$. The set of simple functions is dense in $L_{2}(\lambda)$.

2) If $f, g$ are square $\lambda$-integrable, then the pointwise product function $f g$ is $\lambda$-integrable. Moreover,

$$
\left\|\int_{\Omega} f g d \lambda\right\| \leq\|f\|_{L_{2}(\lambda)}\|g\|_{L_{2}(\lambda)}, \quad \text { and } \quad \sup _{h \in B_{L_{2}(\lambda)}}\left\|\int_{\Omega} h f d \lambda\right\|=\|f\|_{L_{2}(\lambda)} .
$$

3) Suppose that $X=l_{2}$ and $\lambda$ is positive. Then for every $g \in L_{2}(\lambda)$ the expression $\|f\|_{g}:=\left(\left\langle\int_{\Omega} f^{2} d \lambda, \int_{\Omega} g^{2} d \lambda\right\rangle\right)^{\frac{1}{2}}, f \in L_{2}(\lambda)$, defines a seminorm on $L_{2}(\lambda)$.

The statements 1) and 2) are straightforward consequences of the inequalities that can be found at the beginning of Section 3 of [11], and the proof of Proposition 8 and Lemma 9 in [11]. If $\lambda$ is positive, the formula $\mu_{g}(A):=\left\langle\lambda(A), \int_{\Omega} g^{2} d \lambda\right\rangle, A \in \Sigma$, defines a positive (scalar) finite measure, which gives 3$)$. 
From now on, we center our attention in the case of countably additive vector measures with values in $l_{2}$; if $\lambda: \Sigma \rightarrow l_{2}$, a direct calculation shows that the formula $\|f\|_{\lambda}:=\left\|\int_{\Omega} f^{2} d \lambda\right\|^{\frac{1}{2}}, f \in L_{2}(\lambda)$, provides another expression for the norm of $L_{2}(\lambda)$. This formula will be widely used in the rest of the paper.

Let us introduce the space $l_{2}\left(l_{2}\right)$ of 2-summable sequences of elements of $l_{2}$. If $z=\sum_{j=1}^{\infty}\left(\sum_{i=1}^{\infty} \nu_{j i} e_{i}\right) e_{j} \in l_{2}\left(l_{2}\right)$, its norm is given by $\|z\|_{2,2}=$ $\left(\sum_{j=1}^{\infty}\left\|\sum_{i=1}^{\infty} \nu_{j i} e_{i}\right\|^{2}\right)^{\frac{1}{2}}=\left(\sum_{j=1}^{\infty} \sum_{i=1}^{\infty} \nu_{j i}^{2}\right)^{\frac{1}{2}}$.

In the rest of this section we fix $\lambda$ and we assume that it is a positive countably additive vector measure on $l_{2}$. This will simplify the notation, since no explicit reference will be given to $\lambda$ in the following definitions. However, note that all these definitions depend on $\lambda$.

Definition 1.1. A countable set of functions $\mathcal{C}=\left\{h_{i} \in L_{2}(\lambda) \mid i \in N\right\}$ is a $\lambda$-orthonormal system if

1) $\int_{\Omega} h_{i} h_{j} d \lambda=0$ for different $i, j \in N$,

2) $\left\|h_{i}\right\|_{\lambda}=1$ for every $i \in N$, and

3) the expression $T_{\mathcal{C}}(f):=\sum_{i=1}^{\infty}\left(\int_{\Omega} f h_{i} d \lambda\right) e_{i}$ defines a continuous operator $T_{\mathcal{C}}: L_{2}(\lambda) \rightarrow l_{2}\left(l_{2}\right)$.

We will also consider the case of finite $\lambda$-orthonormal systems assuming the obvious restrictions in the definition above. Straightforward calculations show that a $\lambda$-orthonormal system defines a linearly independent set of functions. We denote by $S(\mathcal{C})$ the (closed) subspace generated by $\mathcal{C}$, i.e. the closure of the linear span of $\mathcal{C}$.

Example 1. Let $\left(\Omega, \Sigma, \mu_{0}\right)$ be a positive finite measure space. We define the vector measure $\lambda_{0}: \Sigma \rightarrow l_{2}$ by $\lambda_{0}(A):=\mu(A) e_{1}, A \in \Sigma$. It is easy to see that $L_{2}\left(\mu_{0}\right)=L_{2}\left(\lambda_{0}\right)$. Suppose that $\mathcal{C}_{0}=\left\{f_{i} \mid i \in N\right\}$ is an orthonormal sequence of functions of $L_{2}\left(\mu_{0}\right)$. Then clearly it satisfies the requirements 1 ) and 2) of Definition 1.1. Moreover $\left\|T_{\mathcal{C}_{0}}(g)\right\|_{2,2}=\left(\sum_{i=1}^{\infty}\left|\int_{\Omega} g f_{i} d \mu_{0}\right|^{2}\right)^{\frac{1}{2}} \leq\|g\|_{L_{2}\left(\mu_{0}\right)}$. Therefore, $T_{\mathcal{C}_{0}}$ is a continuous operator and $\mathcal{C}_{0}$ is a $\lambda_{0}$-orthonormal system.

Proposition 1.2. Let $\mathcal{C}=\left\{h_{i} \in L_{2}(\lambda) \mid i \in N\right\}$ be a $\lambda$-orthonormal system. Then $S(\mathcal{C})$ is isomorphic to $l_{2}$. Thus, each function $g \in S(\mathcal{C})$ can be written as a series $g=\sum_{i=1}^{\infty} \alpha_{i} h_{i}$, where $\left(\alpha_{i}\right)_{i=1}^{\infty} \in l_{2}$.

Proof. If $n \in N$ and $g=\sum_{i=1}^{n} \alpha_{i} h_{i},\|g\|_{\lambda}=\left\|\int_{\Omega} \sum_{i=1}^{n} \alpha_{i}^{2} h_{i}^{2} d \lambda\right\|^{\frac{1}{2}} \leq$ $\left(\sum_{i=1}^{n} \alpha_{i}^{2}\right)^{\frac{1}{2}}$, and $\left\|T_{\mathcal{C}}(g)\right\|_{2,2}=\left(\sum_{i=1}^{\infty}\left\|\int_{\Omega} g h_{i} d \lambda\right\|^{2}\right)^{\frac{1}{2}}=\left(\sum_{i=1}^{n} \alpha_{i}^{2}\right)^{\frac{1}{2}} \leq\left\|T_{\mathcal{C}}\right\|$ $\|g\|_{\lambda}$. Therefore, $S(\mathcal{C})$ is isomorphic to $l_{2}$, and each element $f \in S(\mathcal{C})$ can be written as a series $\sum_{i=1}^{\infty} \alpha_{i} h_{i}=\lim _{n} \sum_{i=1}^{n} \alpha_{i} h_{i}$, where $\left(\alpha_{i}\right)_{i=1}^{\infty} \in l_{2}$. 
Lemma 1.1. Let $\mathcal{C}$ be a $\lambda$-orthonormal system. If $g=\sum_{i=1}^{\infty} \alpha_{i} h_{i} \in$ $S(\mathcal{C})$ and $j \in N$, then $\int_{\Omega} g h_{j} d \lambda=\int_{\Omega} \alpha_{j} h_{j}^{2} d \lambda$.

The straightforward proof is a consequence of Proposition 1.1,2) and Proposition 1.2.

Definition 1.2. Let $\mathcal{C}$ be a $\lambda$-orthonormal system. We call the $\lambda$ orthogonal complement of $S(\mathcal{C})$ to $S^{\lambda}(\mathcal{C}):=\left\{f \in L_{2}(\lambda) \mid \int_{\Omega} f g d \lambda=0, g \in\right.$ $S(\mathcal{C})\}$.

Lemma 1.2. Let $\mathcal{C}$ be a $\lambda$-orthonormal system. A function $f \in L_{2}(\lambda)$ belongs to $S^{\lambda}(\mathcal{C})$ if and only if $f$ is $\lambda$-orthogonal to each function $h_{i}$ of $\mathcal{C}$. Moreover, $S^{\lambda}(\mathcal{C})$ is a closed subspace of $L_{2}(\lambda)$.

Proof. Let $f \in L_{2}(\lambda)$ such that $\int_{\Omega} f h_{i} d \lambda=0$ for every $i \in N$, and consider a function $g \in S(\mathcal{C})$. Then there is a sequence of functions $\left(g_{i}\right)_{i=1}^{\infty} \subset$ $\operatorname{span}\left\{h_{i} \mid i \in N\right\}$ that converges to $g$ in $L_{2}(\lambda)$. Then, using the statement 2) of Proposition 1.1 we obtain $\left\|\int_{\Omega} f g d \lambda\right\|=\left\|\int_{\Omega} f g d \lambda-\int_{\Omega} f g_{i} d \lambda\right\| \leq\|f\|_{\lambda}\left\|g-g_{i}\right\|_{\lambda}$ for every $i \in N$. This implies $\int_{\Omega} f g d \lambda=0$. On the other hand, the linearity of the integral $\int_{\Omega} f(\cdot) d \lambda$ for every $f \in L_{2}(\lambda)$ implies that $S^{\lambda}(\mathcal{C})$ is a subspace. A direct argument using the inequalities above shows that $S^{\lambda}(\mathcal{C})$ is also closed.

Definition 1.3. If $\mathcal{C}=\left\{h_{i} \mid i \in N\right\}$ is a $\lambda$-orthonormal system in $L_{2}(\lambda)$ and $f, g \in L_{2}(\lambda)$, we define the $\mathcal{C}$-orthogonal distance between $f$ and $g$ as

$$
d_{\mathcal{C}}(f, g):=\left\|T_{\mathcal{C}}(f-g)\right\|_{2,2} .
$$

The $\mathcal{C}$-orthogonal distance between a subspace $S \subset L_{2}(\lambda)$ and a function $f \in$ $L_{2}(\lambda)$ is

$$
d_{\mathcal{C}}(f, S):=\inf _{g \in S}\left\|T_{\mathcal{C}}(f-g)\right\|_{2,2}
$$

In the following examples we explicitly obtain the best approximation in finite dimensional subspaces $S$ of $L_{2}(\lambda)$ to arbitrary functions $f \in L_{2}(\lambda)$ with respect to particular $\mathcal{C}$-orthogonal distance criteria. Our aim is to show that some usual minimal distance criteria can be written in terms of adequate $\mathcal{C}$ orthogonal distances. This motivates the approximation results given in Theorem 1.1 and Theorem 1.2.

Example 2. Consider the vector measure $\lambda_{0}$ in Example 1 and the orthonormal sequence that defines $\mathcal{C}_{0}$. If $n \in N$, let us define the $\lambda_{0}$-orthonormal system $\mathcal{C}_{n}:=\left\{f_{i} \mid i=1, \ldots, n\right\}$, the $n$ first functions of $\mathcal{C}_{0}$. A direct calculation 
shows that $T_{\mathcal{C}_{n}}(f)=0$ if and only if $f$ is orthogonal to the subspace $S_{n}=S\left(\mathcal{C}_{n}\right)$ of $L_{2}\left(\mu_{0}\right)$. Therefore, the $\mathcal{C}_{n}$-orthogonal distance from a function $g \in L_{2}\left(\lambda_{0}\right)$ to $S_{n}$ is attained for the function $P_{S_{n}}(g)$, where $P_{S}$ is the canonical projection $P_{S_{n}}: L_{2}\left(\mu_{0}\right) \rightarrow S_{n}$.

Example 3. Let $n \in N$ and let $\left(\Omega, \Sigma, \mu_{3}\right)$ be a measure space that satisfies $\mu_{3}(\Omega)=n$ and that there is a measurable partition $\mathcal{A}:=\left\{A_{i} \mid i=\right.$ $1, \ldots, n\}$ of $\Omega$ such that $\mu_{3}\left(A_{i}\right)=1, i=1, \ldots, n$. Consider the positive vector measure $\lambda_{3}: \Sigma \rightarrow l_{2}$ defined by $\lambda_{3}(A):=\sum_{i=1}^{n} \mu_{3}\left(A \cap A_{i}\right) e_{i}, A \in \Sigma$. Consider the set of characteristic functions $\mathcal{C}_{3}=\left\{\chi_{A_{i}} \mid i=1, \ldots, n\right\}$. It is easy to see that $\mathcal{C}_{3}$ defines a $\lambda_{3}$-orthonormal system; in particular, the norms $\left\|\chi_{A_{i}}\right\|_{\lambda_{3}}=1$, since $\mu_{3}\left(A_{i}\right)=1$, for each $i=1, \ldots, n$. A direct calculation shows that $T_{\mathcal{C}_{3}}(f)=0$ if and only if $\int_{A_{i}} f d \mu_{3}=0$ for every $i=1, \ldots, n$. Moreover, if $S=S\left(\mathcal{C}_{3}\right)$ the distance $d_{\mathcal{C}_{3}}(f, S)$ from the space $S$ to a function $f \in L_{2}\left(\lambda_{3}\right)$ is attained for the function $\sum_{i=1}^{n} \alpha_{i} \chi_{A_{i}}$, where $\alpha_{i}:=\int_{A_{i}} f d \mu, i=1, \ldots, n$.

Theorem 1.1. $\quad$ Let $\mathcal{C}$ be a $\lambda$-orthonormal system. Let $g \in L_{2}(\lambda)$. Then the $\mathcal{C}$-orthogonal distance between $g$ and $S(\mathcal{C})$ is attained for the function $P_{\mathcal{C}}(g)$ $:=\sum_{i=1}^{\infty} \beta_{i} h_{i} \in L_{2}(\lambda)$, where

$$
\beta_{i}=\left\langle\int_{\Omega} g h_{i} d \lambda, \int_{\Omega} h_{i}^{2} d \lambda\right\rangle, \quad i \in N .
$$

Moreover, the operator $P_{\mathcal{C}}: L_{2}(\lambda) \rightarrow S(\mathcal{C})$ is a continuous projection on $S(\mathcal{C})$.

Proof. First let us show that $P_{\mathcal{C}}$ is a continuous projection. To prove that it is well defined, let $g$ be a function of $L_{2}(\lambda)$. By Proposition 1.2 it is enough to show that $\left(\beta_{i}\right)_{i=1}^{\infty} \in l_{2}$, where $\beta_{i}=\left\langle\int_{\Omega} g h_{i} d \lambda, \int_{\Omega} h_{i}^{2} d \lambda\right\rangle, i \in N$.

But $\sum_{i=1}^{\infty}\left(\left\langle\int_{\Omega} g h_{i} d \lambda, \int_{\Omega} h_{i}^{2} d \lambda\right\rangle\right)^{2} \leq \sum_{i=1}^{\infty}\left\|\int_{\Omega} g h_{i} d \lambda\right\|^{2} \leq\left\|T_{\mathcal{C}}\right\|^{2}\|g\|_{\lambda}^{2}$. Moreover, $\left\|P_{\mathcal{C}}(g)\right\|_{\lambda}=\left\|\int_{\Omega} \sum_{i=1}^{\infty} \beta_{i}^{2} h_{i}^{2} d \lambda\right\|^{\frac{1}{2}} \leq\left(\sum_{i=1}^{\infty} \beta_{i}^{2}\right)^{\frac{1}{2}} \leq\left\|T_{\mathcal{C}}\right\|\|g\|_{\lambda}$. Thus, $P_{\mathcal{C}}$ is a continuous operator.

Now, let $n \in N$ and consider an arbitrary function $\sum_{i=1}^{n} \alpha_{i} h_{i} \in S(\mathcal{C})$. A direct calculation gives

$$
\begin{aligned}
& \left\|T_{\mathcal{C}}\left(g-\sum_{i=1}^{n} \alpha_{i} h_{i}\right)\right\|_{2,2}^{2}=\sum_{i=1}^{n}\left(\left\langle\int_{\Omega} g h_{i} d \lambda, \int_{\Omega} g h_{i} d \lambda\right\rangle\right. \\
& \left.-2 \alpha_{i}\left\langle\int_{\Omega} g h_{i} d \lambda, \int_{\Omega} h_{i}^{2} d \lambda\right\rangle+\alpha_{i}^{2}\right)+\sum_{i=n+1}^{\infty}\left\|\int_{\Omega} g h_{i} d \lambda\right\|^{2} .
\end{aligned}
$$


Note that the second term of the expression above is finite, since $T_{\mathcal{C}}$ is continuous. The equations

$$
\frac{\partial\left\|T_{\mathcal{C}}\left(g-\sum_{i=1}^{n} \alpha_{i} h_{i}\right)\right\|_{2,2}^{2}}{\partial \alpha_{i}}=-2\left\langle\int_{\Omega} g h_{i} d \lambda, \int_{\Omega} h_{i}^{2} d \lambda\right\rangle+2 \alpha_{i}=0,
$$

$i=1, \ldots, n$, give the result. Note that the same result holds for every $n \in N$, and then the sequence $\left(\beta_{i}\right)_{i=1}^{\infty}$ defines the minimum when we consider the whole space $S(\mathcal{C})$ as a consequence of the sectional convergence that provides the isomorphy between $l_{2}$ and this space (Proposition 1.2).

Definition 1.4. Let $\mathcal{C}$ be a $\lambda$-orthonormal system. Let $g \in L_{2}(\lambda)$ and let $P_{\mathcal{C}}$ be as in the theorem above. We call $P_{\mathcal{C}}(g)$ the self-weighted approximation of the subspace $S(\mathcal{C})$ to the function $g$ with respect to the positive vector measure $\lambda$.

Theorem 1.2. $\quad$ Let $\mathcal{C}$ be a $\lambda$-orthonormal system and let $g \in L_{2}(\lambda)$. Then $g-P_{\mathcal{C}}(g) \in S^{\lambda}(\mathcal{C})$ if and only if $\left\|T_{\mathcal{C}}\left(g-P_{\mathcal{C}}(g)\right)\right\|_{2,2}=0$. Moreover, in this case $P_{\mathcal{C}}(g)$ is the function of $S(\mathcal{C})$ for which the infimum $\inf _{f \in S(\mathcal{C})}\|g-f\|_{\lambda}$ is attained.

Proof. Note that the first equivalence is obvious, since $T_{\mathcal{C}}\left(g-P_{\mathcal{C}}(g)\right)=0$ if and only if $g-P_{\mathcal{C}}(g) \in S^{\lambda}(\mathcal{C})$, as a consequence of Lemma 1.2. Now let us calculate the $\inf _{f \in S(\mathcal{C})}\|g-f\|_{\lambda}$ in the case that $g_{0}:=g-P_{\mathcal{C}}(g) \in S^{\lambda}(\mathcal{C})$. Consider the representation of $g=P_{\mathcal{C}}(g)+g_{0}$. For every function $\sum_{i=1}^{\infty} \alpha_{i} h_{i} \in$ $S(\mathcal{C})$, using of the $\lambda$-orthogonality of the functions of $\mathcal{C}$, we obtain

$$
\begin{aligned}
& \left\|g-\sum_{i=1}^{\infty} \alpha_{i} h_{i}\right\|_{\lambda}^{2}=\left\|\int_{\Omega} g_{0}^{2} d \lambda\right\|^{2}+2 \sum_{i=1}^{\infty}\left(\alpha_{i}-\beta_{i}\right)^{2}\left\langle\int_{\Omega} h_{i}^{2} d \lambda, \int_{\Omega} g_{0}^{2} d \lambda\right\rangle \\
& +\sum_{i=1}^{\infty} \sum_{k=1}^{\infty}\left(\alpha_{i}-\beta_{i}\right)^{2}\left(\alpha_{k}-\beta_{k}\right)^{2}\left\langle\int_{\Omega} h_{i}^{2} d \lambda, \int_{\Omega} h_{k}^{2} d \lambda\right\rangle .
\end{aligned}
$$

Clearly, this expression attains its minimum value $\left\|\int_{\Omega} g_{0}^{2} d \lambda\right\|^{2}$ if $\beta_{i}=\alpha_{i}$ for every $i \in N$.

The theorem above establishes the relation between $\lambda$-orthogonality and best approximation in our vector integration setting. Note that this result is similar to the one that holds for positive finite (scalar) measures $\mu$, but in this 
case the space can always we written as a direct sum of $S(\mathcal{C})$ and its orthogonal space $S^{\mu}(\mathcal{C})$. However, this is not true in our context. This motivates the definition of self-weighted approximation taking into account the verification of certain vector orthogonality conditions. In the following section we provide a geometrical motivation of its properties.

\section{§2. The Geometric Properties of the Self-weighted Function Approximation}

In this section we introduce the notion of conic vector measure and we show that it is possible to explain the properties of the self-weighted approximation in terms of a direct geometrical argument. Our aim is to study from this point of view the structure of the coefficients $\beta_{i}$ that appear in the projection $P_{\mathcal{C}}(g)$ of a function $g \in L_{2}(\lambda)$.

Definition 2.1. Let $\lambda: \Sigma \rightarrow l_{2}$ be a countably additive vector measure, and let $\theta=\left(\sigma_{i}\right)_{i=1}^{\infty}$ be a sequence of elements $\sigma_{i} \in\{1,-1\}$. Consider the cone $C_{\theta}$ generated by $\left\{\sigma_{i} e_{i} \mid i \in N\right\}$, i.e. the closure of the set of positive linear combinations of the elements of this set. We say that $\lambda$ is a $\theta$-conic measure if $r g(\lambda) \subset C_{\theta}$, and there is a family of measurable subsets $\left\{A_{i} \mid i \in N\right\}$ such that $\lambda\left(A_{i}\right) \neq 0$ and $\lambda\left(A_{i}\right) \in\left\langle e_{i}\right\rangle$ for every $i \in N$, and $\cup_{i=1}^{\infty} A_{i}=\Omega$.

We will simply say that $\lambda$ is a conic measure if it is a $\theta$-conic measure for a certain sequence $\theta$. If $\theta$ is the sequence defined by $\sigma_{i}=1$ for every $i \in N$, we will say that $\lambda$ is a positive conic measure.

Proposition 2.1. $\quad$ Let $\lambda$ be a conic measure and let $\mu$ be a Rybakov measure for $\lambda$. Then the associated family of measurable subsets $\left\{A_{i} \mid i \in N\right\}$ defines ( $\mu$-a.e.) a partition of $\Omega$, and $\lambda(A):=\sum_{i=1}^{\infty}\left\langle\lambda\left(A \cap A_{i}\right), e_{i}\right\rangle e_{i}, A \in \Sigma$. Consequently, there is a Bochner integrable function $\phi \in L_{1}\left(\mu, l_{2}\right)$ such that $\lambda(A):=\int_{A} \phi d \mu, A \in \Sigma$.

Proof. First we show that for each $i \in N$ and every measurable subset $B \subset A_{i}, \lambda(B) \in\left\langle e_{i}\right\rangle$. Consider the sequence $\theta=\left(\sigma_{i}\right)_{i=1}^{\infty}$ in the definition of the measure $\lambda$. Fix $i \in N$. If there is a measurable set $B \subset A_{i}$ which does not satisfy the property, we can find an index $j \neq i$ such that $\left\langle\lambda(B), e_{j}\right\rangle \neq 0$. We can assume without loss of generality that $\sigma_{j}=1$. Thus, the measure $\lambda_{e_{j}}$ defined by $\lambda_{e_{j}}(A):=\left\langle\lambda, e_{j}\right\rangle(A), A \in \Sigma$, is positive, and then $\left\langle\lambda, e_{j}\right\rangle\left(A_{i}\right) \geq\left\langle\lambda, e_{j}\right\rangle(B)>0$, a contradiction. Thus, if for $i \neq j, B \subset A_{i} \cap A_{j}$, and $\mu$ is a Rybakov measure for $\lambda$ we have that $\mu(B)=0$. 
Now, if $i \in N$, it is clear that $\mu$ controls $\lambda_{e_{i}}$, and then the Radon-Nikodym Theorem gives a positive function $f_{i}$ if $\sigma_{i}=1$ (negative if $\sigma_{i}=-1$ ) with support in $A_{i}$ such that $\lambda_{e_{i}}(A)=\int_{A} f_{i} d \mu$. Let $\phi$ the pointwise defined function $\phi:=\sum_{i=1}^{\infty} f_{i} e_{i}$. Then the countably additivity of $\lambda$ gives the convergence of the sequence $\left(\sum_{i=1}^{n} f_{i} e_{i}\right)_{n=1}^{\infty}$ in $L_{1}\left(\mu, l_{2}\right)$ to $\phi$, and $\lambda(A)=\int_{A} \phi d \mu, A \in \Sigma$.

Corollary 2.1. In the conditions of Proposition 2.1, the equality

$$
\int_{A} f d \lambda=\sum_{i=1}^{\infty}\left(\int_{A_{i} \cap A} f d\left\langle\left.\lambda\right|_{A_{i}}, e_{i}\right\rangle\right) e_{i}
$$

holds for every function $f \in L_{1}(\lambda)$ and every $A \in \Sigma$.

Corollary 2.2. $\quad$ Let $\lambda$ be a conic measure. Then there is a positive conic measure $\lambda^{\prime}$ such that $L_{2}(\lambda)=L_{2}\left(\lambda^{\prime}\right)$ isometrically.

For the proof of this corollary is enough to consider the vector measure $\lambda^{\prime}$ defined by the function $|\phi|$, where $\phi$ is the Bochner integrable function of Proposition 2.1. A direct calculation shows that $\|g\|_{\lambda}=\|g\|_{\lambda^{\prime}}$ for every $g \in$ $L_{2}(\lambda)$. Therefore, from now on we can assume without loss of generality that $\lambda$ is a positive conic measure.

Proposition 2.2. $\quad$ Consider a positive conic measure $\lambda$ and a finite $\lambda$ orthonormal system $\mathcal{C}:=\left\{h_{i} \in L_{2}(\lambda) \mid i=1, \ldots, n\right\}$. Let $h:=\sum_{i=1}^{n} h_{i} \in L_{2}(\lambda)$. Then

$$
\frac{\left(\sum_{i=1}^{n}\left\|h_{i}\right\|_{\lambda}^{4}\right)^{\frac{1}{2}}}{\|h\|_{\lambda}} \leq\left\|T_{\mathcal{C}}\right\| \leq\|h\|_{\lambda} .
$$

Therefore, $\left\|T_{\mathcal{C}}\right\|=\|h\|_{\lambda}$ if $\left(\int_{\Omega} h_{i}^{2} d \lambda\right)_{i=1}^{n}$ is an orthogonal sequence of $l_{2}$.

Proof. By Proposition 2.1, there is a partition $\left\{A_{j} \mid j \in N\right\}$ of $\Omega$ such that $\lambda(A)=\sum_{j=1}^{\infty} \mu_{j}(A) e_{j}$, where $\mu_{j}(A):=\left\langle\lambda\left(A \cap A_{j}\right), e_{j}\right\rangle, A \in \Sigma$. The sequence $\left(h_{i}\right)_{i=1}^{n}$ is orthogonal in all the Hilbert spaces $L_{2}\left(A_{j}, \mu_{j}\right), j \in N$. Then $\left\langle\int_{A_{j}} h^{2} d \lambda, e_{j}\right\rangle=\int_{A_{j}} \sum_{i=1}^{n} h_{i}^{2} d \mu_{j}$ for every $j \in N$. If $g \in L_{2}(\lambda)$, for every $h_{i}$ the function $g h_{i} \in L_{1}(\lambda)$ (Proposition 1.1), and $\int_{\Omega} g h_{i} d \lambda=\sum_{j=1}^{\infty}\left(\int_{A_{j}} g h_{i} d \mu_{j}\right) e_{j}$ as a consequence of Corollary 2.1. Then $\left\|T_{\mathcal{C}}(g)\right\|_{2,2}=\left(\sum_{i=1}^{n}\left(\sum_{j=1}^{\infty}\left(\int_{A_{j}} g h_{i}\right.\right.\right.$ $\left.\left.\left(\mu_{j}\right)^{2}\right)\right)^{\frac{1}{2}}$. Applying the Hölder inequality for the measures $\mu_{j}$ and taking into 
account the properties of the norm of $l_{2}$, we obtain

$$
\begin{aligned}
& \left\|T_{\mathcal{C}}(g)\right\|_{2,2} \leq\left(\sum_{i=1}^{n}\left(\sum_{j=1}^{\infty}\left(\int_{A_{j}} g^{2} d \mu_{j}\right)\left(\int_{A_{j}} h_{i}^{2} d \mu_{j}\right)\right)\right)^{\frac{1}{2}} \\
& =\left(\sum_{j=1}^{\infty}\left(\int_{A_{j}} g^{2} d \mu_{j}\right)\left(\sum_{i=1}^{n} \int_{A_{j}} h_{i}^{2} d \mu_{j}\right)\right)^{\frac{1}{2}}=\mid\left\langle\int_{\Omega} g^{2} d \lambda, \int_{\Omega} \sum_{i=1}^{n} h_{i}^{2} d \lambda\right\rangle^{\frac{1}{2}} .
\end{aligned}
$$

Then $\left\|T_{\mathcal{C}}\right\| \leq\|h\|_{\lambda}$. Moreover, if we consider the function $\frac{h}{\|h\|_{\lambda}}$, we obtain

$$
\left\|T_{\mathcal{C}}\left(\frac{h}{\|h\|_{\lambda}}\right)\right\|_{2,2}=\frac{\left\|\sum_{i=1}^{n}\left(\int_{\Omega} h_{i} h d \lambda\right) e_{i}\right\|_{2,2}}{\|h\|_{\lambda}}=\frac{\left(\sum_{i=1}^{n}\left\|\int_{\Omega} h_{i}^{2} d \lambda\right\|^{2}\right)^{\frac{1}{2}}}{\|h\|_{\lambda}} \leq\left\|T_{\mathcal{C}}\right\|
$$

Using the last arguments we can provide a geometrical interpretation of the self-weighted approximation. Consider a positive conic measure $\lambda$ and a $\lambda$-orthogonal system $\mathcal{C}=\left\{h_{i} \mid i \in N\right\}$. Let $g \in L_{2}(\lambda)$. Then we have shown in Section 1 that $P_{\mathcal{C}}(g)=\sum_{i=1}^{\infty} \beta_{i} h_{i}$ is a function of $S(\mathcal{C})$. If $i \in N$, we have $\beta_{i}=\sum_{j=1}^{\infty}\left(\int_{A_{j}} g h_{i} d\left\langle\lambda, e_{j}\right\rangle\right)\left(\int_{A_{j}} h_{i}^{2} d\left\langle\lambda, e_{j}\right\rangle\right)$. Thus, if $\beta_{i} \neq 0$, we can define

$$
\operatorname{Cos}\left(\gamma_{i}\right):=\left\langle\frac{\int_{\Omega} g h_{i} d \lambda}{\left\|\int_{\Omega} g h_{i} d \lambda\right\|}, \int_{\Omega} h_{i}^{2} d \lambda\right\rangle
$$

Therefore, we obtain a representation of $\beta_{i}$ as a product of an scalar term $\left\|\int_{\Omega} g h_{i} d \lambda\right\|$ and an angular term $\operatorname{Cos}\left(\gamma_{i}\right)$, since $\beta_{i}=\left\|\int_{\Omega} g h_{i} d \lambda\right\| \operatorname{Cos}\left(\gamma_{i}\right)$. Note that $\operatorname{Cos}\left(\gamma_{i}\right)$ is either 1 or -1 when $\int_{\Omega} g h_{i} d \lambda \in\left\langle\int_{\Omega} h_{i}^{2} d \lambda\right\rangle$. Thus, $\left|\beta_{i}\right|$ is weighted by the term $\operatorname{Cos}\left(\gamma_{i}\right)$, that represents the degree of coincidence between the directions defined by the vectors $\left(\int_{A_{j}} g h_{i} d\left\langle\lambda, e_{j}\right\rangle\right)_{j=1}^{\infty}$ and $\left(\int_{A_{j}} h_{i}^{2} d\left\langle\lambda, e_{j}\right\rangle\right)_{j=1}^{\infty}$. In particular, if $g$ verifies that $g-P_{\mathcal{C}}(g) \in S^{\lambda}(\mathcal{C})$ we obtain that $\operatorname{Cos}\left(\gamma_{i}\right)$ is either 1 or -1 . Of course, there is no angular contribution in the classical case of finite positive (scalar) measures.

Let us finish the paper with some examples. In the first one, we show a function $g$ that satisfies that the self-weighted approximation $P_{\mathcal{C}}(g)$ is similar to the one obtained with the classical Hilbert space approximation.

Example 4. Consider the usual Lebesgue measure space $([0,3], \Sigma, \nu)$ and the vector measure $\lambda_{4}: \Sigma \rightarrow l_{2}^{3}$ given by $\lambda_{4}(A):=\sum_{i=1}^{3} \nu(A \cap[i-1, i]) e_{i}$. 
Let us define the set of functions $C:=\left\{h_{1}(x), h_{2}(x), h_{3}(x)\right\}$ on $[0,3]$ by

$$
\begin{aligned}
h_{1}(x) & :=1 \\
h_{2}(x) & :=\frac{3}{2}-\frac{11}{2} x+\frac{9}{2} x^{2}-x^{3} \\
h_{3}(x) & :=\frac{171}{175}-\frac{63}{5} x+\frac{393}{10} x^{2}-\frac{252}{5} x^{3}+\frac{309}{10} x^{4}-9 x^{5}+x^{6} .
\end{aligned}
$$

The set $\mathcal{C}_{4}:=\left\{\frac{h_{i}}{\left\|h_{i}\right\|_{\lambda_{4}}} \mid i=1,2,3\right\}$ is a $\lambda_{4}$-orthonormal system. Consider the function $g(x):=\exp \left(-(x-2.2)^{2}\right)+1.5 \exp (-x)$. The projections of $g(x)$ using our procedure and the usual Hilbert space formulae are

$$
P(g)(x):=P_{\mathcal{C}_{4}}(g)(x)=0.989188 h_{1}(x)+0.332633 h_{2}(x)+0.003089 h_{3}(x),
$$

and

$$
H(g)(x)=0.989188 h_{1}(x)+0.338121 h_{2}(x)+0.007486 h_{3}(x),
$$

respectively. Thus, Figure 1 shows two similar approximations to the function $g(x)$.

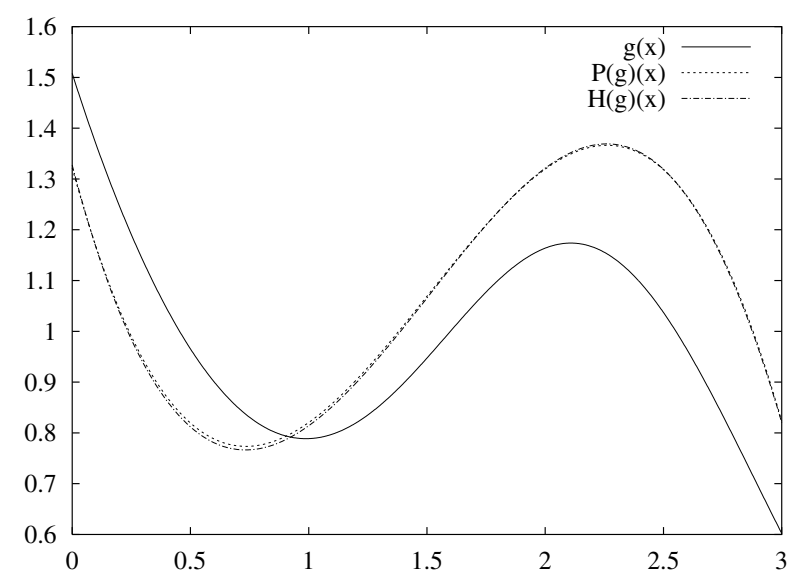

Figure 1.- Functions $g(x)=\exp \left(-(x-2.2)^{2}\right)+1.5 \exp (-x), P(g)(x)$ and

$$
H(g)(x) \text {. }
$$

Although neither $P(g)$ nor $H(g)$ provide good approximations to the function $g$, the behaviour of both approximations is quite similar. From the geometrical point of view, this can be explained by the fact that the distribution of the integrals $\left(\int_{[j-1, j]} g \frac{h_{i}}{\left\|h_{i}\right\|_{\lambda_{4}}} d\left\langle\lambda_{4}, e_{j}\right\rangle\right)_{j=1}^{3}$ is equivalent to the one of the integrals $\left(\int_{[j-1, j]}\left(\frac{h_{i}}{\left\|h_{i}\right\|_{\lambda_{4}}}\right)^{2} d\left\langle\lambda_{4}, e_{j}\right\rangle\right)_{j=1}^{3}$ for each $i=1,2,3$. 
However, the situation shown in this example changes when we want to approximate a signal whose distribution of the integrals of $g h_{i}$ on the sets $A_{j}$ is equivalent to the distribution of only one of the functions of $\mathcal{C}$. In this case, the fit gives more weight to this function, as the following example shows.

Example 5. Let $([0,2], \Sigma, \nu)$ be the usual Lebesgue measure space. Consider the function $g(x):=e^{-x} \chi_{[0,1]}$. Suppose that $g(x)$ is a signal which we know by theoretical arguments that must be close to an exponential function on $[0,2]$, but we only know it in the interval $[0,1]$.

A right solution to this problem would be given by an extrapolation procedure using the function $h_{1}(x):=e^{-x}$ as an element of the basis. Our theoretical framework provides an approximation technique taking into account this fact. Consider the vector measure $\lambda_{5}: \Sigma \rightarrow l_{2}^{2}$ defined by $\lambda_{5}(A):=\nu([0,1] \cap A) e_{1}+$ $\nu([1,2] \cap A) e_{2}$ and the $\lambda_{5}$-orthonormal system $\mathcal{C}_{5}:=\left\{\frac{h_{1}}{\left\|h_{1}\right\|_{\lambda_{5}}}, \frac{h_{2}}{\left\|h_{2}\right\|_{\lambda_{5}}}\right.$, where

$$
h_{1}(x):=e^{-x}, \quad \text { and } \quad h_{2}(x):=0.513440-1.836047 x+x^{2} .
$$

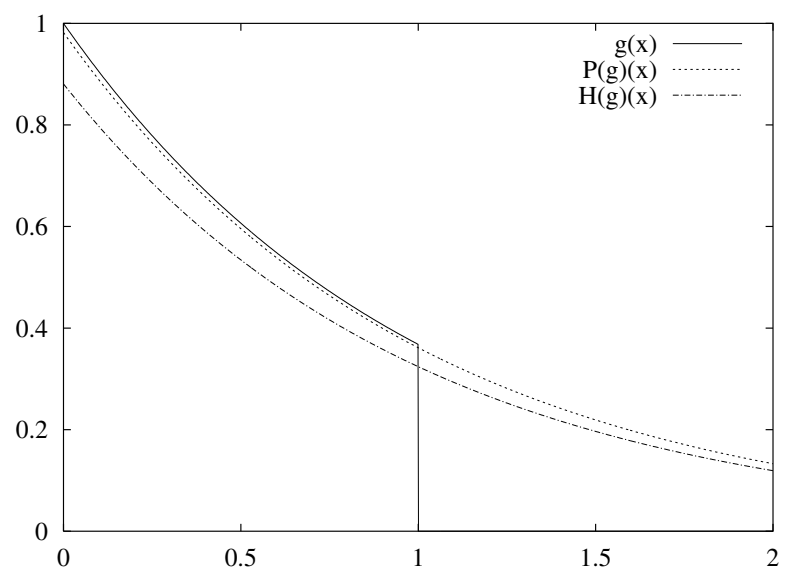

Figure 2.- Functions $g(x)=e^{-x} \chi_{[0,1]}, P(g)(x)$ and $H(g)(x)$.

The self-weighted approximation leads to the projection, $P(g)(x):=P_{\mathcal{C}_{5}}(g)$ $(x)=0.982014 h_{1}(x)$, while the Hilbert space approximation in $[0,2]$ gives $H(g)(x)=0.880797 h_{1}(x)$. As the reader can see, the coefficient of the projection of $g$ with respect to the function $e^{-x}$ is close to 1 in $P(g)$, but this is not the case in $H(g)$. This is caused by the fact that the first function of $\mathcal{C}_{5}$ is self-weighted in the calculus of the projection, and then the fit of the incomplete signal $g$ is better than the usual Hilbert space projection from this 
extrapolation point of view. The fact that the support of the function $\left(e^{-x}\right)^{2}$ is bigger in the interval $[0,1]$ than in $[1,2]$ is reflected in the weight that it induces in the calculation of the coefficient $\beta_{1}$ (see Figure 2).

\section{References}

[1] Curbera, G. P., Banach space properties of $L^{1}$ of a vector measure, Proc. Amer. Math. Soc., 123 (1995), 3797-3806.

[2] Diestel, J. and Uhl, J. J., Vector Measures, Math. Surveys, vol. 15, Amer. Math. Soc., Providence, RI, 1977.

[3] García-Raffi, L. M., Ginestar, D. and Sánchez Pérez, E. A., Integration with respect to a vector measure and function approximation, Abstract and Applied Analysis, 5, 4 (2000), 207-226.

[4] Kluvánek, I. and Knowles, G., Vector Measures and Control Systems, North-Holland, Amsterdam, 1975.

[5] Lacey, H. E., The isometric theory of classical Banach spaces, Springer, Berlin, 1974.

[6] Lewis, D. R., Integration with respect to vector measures, Pacific J. Math., 33 (1970), 157-165.

[7] $\_$On integrability and summability in vector spaces, Illinois J. Math., 16 (1972), 294-307.

[8] Lindenstrauss, J. and Tzafriri, L., Classical Banach Spaces II, Springer-Verlag, Berlin, 1979.

[9] Okada, S. and Ricker, W. J., The range of the integration map of a vector measure, Arch. Math., 64 (1995), 512-522.

[10] Oltra, S., Sánchez Pérez, E. A. and Valero, O., Spaces $L_{2}(\lambda)$ of a positive vector measure $\lambda$ and generalized Fourier coefficients, Rocky Mountain J. Math. to appear.

[11] Sánchez Pérez, E. A., Compactness arguments for spaces of $p$-integrable functions with respect to a vector measure and factorization of operators through Lebesgue-Bochner spaces, Illinois J. Math., 45-3 (2001), 907-923.

[12] Spaces of integrable functions with respect to vector measures of convex range and factorization of operators from $L_{p}$-spaces, Pacific J. Math., 207-2 (2002), 489-495.

[13] , Vector measure orthonormal functions and best approximation for the 4-norm, Arch. Math., 80 (2003), 177-190. 